Check for updates

Cite this: RSC Adv., 2017, 7, 39617

\title{
Influence of ambient conditions on the evolution of wettability properties of an IR-, ns-laser textured aluminium alloy
}

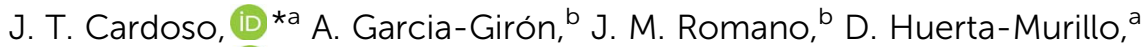 \\ R. Jagdheesh, (D) ${ }^{a}$ M. Walker, ${ }^{c}$ S. S. Dimov ${ }^{b}$ and J. L. Ocaña ${ }^{a}$
}

\begin{abstract}
Micro cell structures of different sizes were patterned using a nanosecond near-infrared laser source on Al2024 aluminium alloy plates with $2 \mathrm{~mm}$ thickness. The influence of laser parameters on the shape and size of the produced patterns were studied together with the evolution of wettability properties over time for different storage conditions. Samples were found to be superhydrophobic from a single step laser patterning, requiring no further treatment. Exposure to ambient air was shown to be a key factor in the property changes of the samples over time. The produced surface patterns with different laser parameter settings were correlated with the contact angle measurements, revealing a great influence of the amount of recast material on the hydrophobic properties. X-Ray photoelectron spectroscopy was used to study the impact of surface chemistry changes on hydrophobicity, analysis of elemental composition proved that chemisorbed organic molecules present in the ambient air were responsible for the hydrophilic to superhydrophobic transition.
\end{abstract}

Received 5th July 2017

Accepted 6th August 2017

DOI: $10.1039 / c 7 r a 07421 b$

rsc.li/rsc-advances addition, the use of chemical compounds results in detritus that are not biodegradable. Therefore, it requires a process to manage the residual compounds. Moreover, the use of chemical reactants requires adequate working conditions since some compounds could be hazardous to humans.

The present work is focused on short pulsed laser machining, ${ }^{18}$ which is an effective and fast solution to produce hydrophobic surfaces with robust micro roughness. ${ }^{\mathbf{1}}$ The ability to promptly form hard textures, turns laser processing into an efficient technique to produce topographies, without requiring additional steps to obtain highly hydrophobic surfaces. The thermal laser-induced surface damages, resulted from splashes and recast materials, are usually minimized or avoided by using ultra-short pulse lasers. Therefore, increasing capital investment, as well as production time.

Recently, patterns composed of micro channels and micro pillars processed in aluminium foils with UV laser sources were reported to be highly hydrophobic. ${ }^{5}$ The present study consists in the one-step fabrication of the micro cells reported in the article, composed of patterned aluminium alloys (Al2024) using a near-infrared (1064 nm) and 15 ns-laser pulses. Combining pulse repetition rate and scanning speed, the pulse trains shape the material's surface, thus creating consecutive holes or trenches. ${ }^{19}$ The thermal laser-material interaction is the key to fabricate such patterns, since an amount of material is vaporized, and the remaining melting pool assembles below and above the surface. Following the principle reported by Jagdheesh et al. ${ }^{5}$ laser processing a succession of lines intersecting themselves at $90^{\circ}$, creates square patterns that will further act
${ }^{a}$ UPM Laser Centre, Polytechnical University of Madrid, Ctra. Valencia, km. 7.3, 28031 Madrid, Spain. E-mail: jose.cardoso@upm.es

${ }^{b}$ School of Mechanical Engineering, University of Birmingham, Edgbaston, Birmingham, B15 2TT, UK

${ }^{c}$ Department of Physics, University of Warwick, Coventry, CV4 7AL, UK 
like air pockets changing the contact angle of water droplets on the processed surface. As a result from the air trapping effect caused by the cell structure, and due to the formation of microcells, the contact with water droplets is made primarily by the created microwalls, decreasing the fraction of area that interacts directly with the drop. Furthermore, it was also reported the presence of smaller particles on the outer part of the laser produced microwalls on aluminium. ${ }^{5}$ These capillarities are also hydrophobic, and help to pin the water droplet on the top part of the walls by holding it with their sub-micron features. By combining these features a transition from Wenzel to Cassie-Baxter ${ }^{20,21}$ state occurs. The droplet contact with the surface is made through an air interface, which lowers the surface tension, and consequently increases the contact angle. Nevertheless, the laser-textured surface exhibited a clear change in wettability in the course of time, from highly hydrophilic right after laser-processing to superhydrophobic after a number of days. ${ }^{22}$ Several hypothesis have been proposed to explain this variation in wettability. For instance, the decomposition of $\mathrm{CO}_{2}$ into carbon ${ }^{10}$ or organic molecules adsorption. ${ }^{23}$

Contact angle values depend on different factors such as pressure, ${ }^{24}$ temperature, ${ }^{25}$ surface roughness, ${ }^{26}$ liquid $^{27}$ and surface chemical compositions. ${ }^{28,29}$ Water droplets adopt the shape which has minimum energy on the contact area. As described by the Wenzel state,$^{30}$ contact angle increases with surface roughness. When microstructures have appropriate shape and size, air trapping is achieved between the liquid and solid interface, causing a decrease in the surface energy, allowing for superhydrophobic properties to be obtained.

The aging process is greatly dependent on sample storage conditions,$^{22}$ laser processing ${ }^{5}$ and the nature of the material itself. $^{28}$ The wettability properties are commonly assessed by measuring the static contact angle. A theta optical measurement system was used to measure the shape of water droplets on the surface. If a given sample is hydrophilic, the droplet spreads $\left(\mathrm{CA}<90^{\circ}\right)$. If it is hydrophobic, the droplet remains with a spherical shape $\left(\mathrm{CA}>90^{\circ}\right)$. In addition, it is superhydrophobic $\left(\mathrm{CA}>150^{\circ}\right)$ or ultrahydrophobic, when droplets have a $180^{\circ}$ contact angle with the surface. For a SCA with a value near $180^{\circ}$, properties such as self-cleaning and anti-icing aid to further increase the industry value on these samples.

The correlation between fluence, scan speed and repetition rate on hydrophobic properties has been previously reported by several authors. ${ }^{1,10,31}$ However there are few reports that study this relation with nanosecond lasers. Sharp et al. ${ }^{32}$ recently reported a study on laser ablation with a ns- IR-laser in which they were not able to identify significant correlation between laser parameters and contact angle, therefore not defining the mechanisms in play that create the hydrophobicity. As this is not the scope of this work, further research is suggested to look for the surface chemistry influence of each factor in time independently for samples patterned with nanosecond lasers.

This work will demonstrate the influence of direct lasertexturing on the wettability of Al2024 plates as a function of time, for samples kept in different conditions, while explaining the influence of varying laser parameters through the analysis of laser accumulated fluence and consequently recast area.

\section{Experimental}

\section{Material, sample preparation and conditioning}

Plates of $75 \mathrm{~mm} \times 75 \mathrm{~mm} \times 2 \mathrm{~mm}$ size of a standard aerospace aluminium alloy, Al2024, were used with an initial surface roughness of $R_{\mathrm{a}}=603 \mathrm{~nm}$, which is considered sufficient for the experiments performed during the project, as the microcell structures that were meant to be produced have several micrometers of height. Therefore, and in order to stay close to industrial requirements, no surface polishing was effectuated. Potential localized scratches and other surface defects previous to the laser processing were ignored, as they did not impact the general laser processing. The performed laser patterns were the only visible structures on the surface after processing and covered any micro scratch that was present, thus the resulting recast from the ablation process is considered to be primarily in contact with the water droplets during SCA measurements. Before the laser process, $\mathrm{Al} 2024$ samples were cleaned using isopropyl alcohol (I.P.A.). In order to remove any powder particles that may had result from the laser patterning, posterior to processing, samples were cleaned by blowing compressed dry air. Immediately after, samples were directly kept in three distinct storage conditions, exposed to ambient air, inside polyethylene $\left(\mathrm{C}_{2} \mathrm{H}_{4}\right)_{n}$ bags and inside polystyrene $\left(\mathrm{C}_{8} \mathrm{H}_{8}\right)_{n}$ boxes, all subjected to lab conditions, under atmospheric pressure and room temperature.

\section{Laser set-up}

The samples were processed using a Yb-doped fiber nanosecond (15 ns) laser source (SPI G4 50W S-type) with an average power $(P)$ of $50 \mathrm{~W}$, a central wavelength in the near-infrared domain $(1064 \mathrm{~nm})$ and a repetition rate up to $1 \mathrm{MHz}$. The beam delivery system integrated a 3D scan head (RhoThor RTA) and a $100 \mathrm{~mm}$ telecentric lens. The laser beam had a Gaussian spatial and temporal profiles and a spot diameter of approximately $35 \mu \mathrm{m}$ at focus. The maximum average power at the end of the optical path was measured at $36 \mathrm{~W}$ using a laser power meter (Gentec-EO UNO). The experiments were performed under atmospheric conditions with the assistance of a fume extractor. Fig. 1 displays a representation of the optical components present in the laser integrated system.

\section{Laser processing}

The scanning process included cross hatching surfaces patterned by laser ablating in two perpendicular directions with the laser at focus. To efficiently form microcells, surrounding channels were patterned in a single scan.

Initially, an array of $25 \mathrm{~mm}^{2}$ fields was textured on $\mathrm{Al} 2024$ plates with a fixed repetition rate of $100 \mathrm{kHz}$ and a hatch distance of $100 \mu \mathrm{m}$. At this frequency, the highest fluence used was $3.21 \mathrm{~J} \mathrm{~cm}^{-2}$ and the scanning speed was varied from 5 to $1000 \mathrm{~mm} \mathrm{~s}^{-1}$, achieving a pulse overlap from 64 to $99.8 \%$ while the pulse energy was varied from 7 to $31 \mu \mathrm{J}$.

Preliminary tests found that pulse energies below $11 \mu \mathrm{J}$ did not result in any surface texturing. With high pulse energy but lower pulse overlap, the single pass laser processing resulted in 


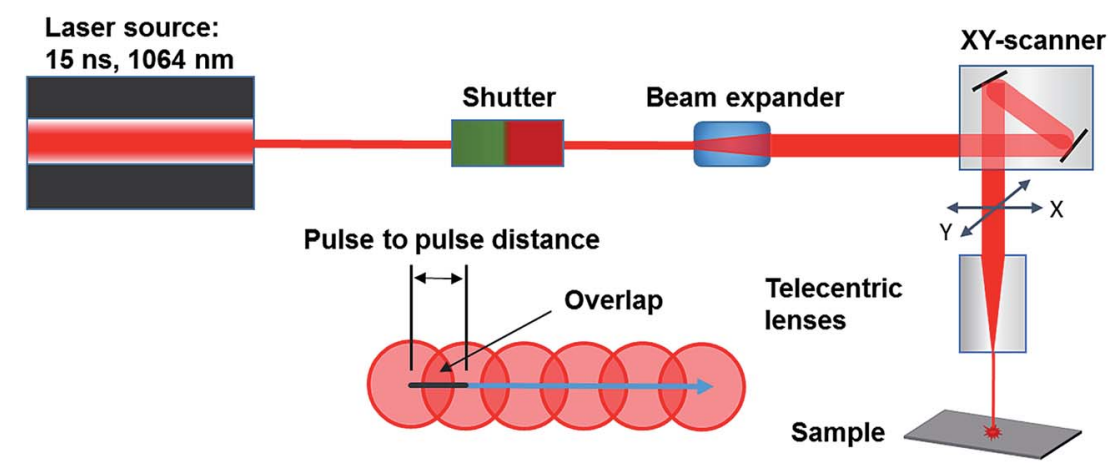

Fig. 1 Integrated laser system representation and an illustrative representation of pulse overlap.

Table 1 Process parameters

Average laser power, $P_{\mathrm{L}}(\mathrm{W})$
Laser spot diameter, $D(\mu \mathrm{m})$
Laser fluence, $F\left(\mathrm{~J} \mathrm{~cm}^{-2}\right)$
Pulse energy, $E(\mu \mathrm{J})$
Repetition rate, $f_{\mathrm{p}}(\mathrm{kHz})$
Hatch distance, $\mathrm{HD}(\mu \mathrm{m})$
Scan speed, $V\left(\mathrm{~mm} \mathrm{~s}^{-1}\right)$

channels, however with insignificant depth below $2 \mu \mathrm{m}$. Then, an increase of the pulse overlap led to increased thermal effects and the formation of a recast layer along the edges of the channels, forming micro pillars along them. These recast formations were particularly important to generate the desired topography. Therefore, a narrow processing window was identified in order to produce these textures, i.e. to combine well defined holes, forming channels, with recasts at their edges by using a high pulse overlap with relatively low pulse energy.

The processing parameters in Table 1 were used to fabricate periodic and homogeneous micro cells. The maximum micro cells' peak to bottom height obtained in these preliminary trials was $16 \mu \mathrm{m}$.

\section{Characterization}

In order to evaluate the topographies, the processed surfaces were analyzed using an Alicona G5 focus variation microscope (FVM), a Leica 3D confocal laser scanning microscope (CLSM), and a HITACHI S-3000N scanning electron microscopy (SEM). The X-ray photoelectron spectroscopy (XPS) data were collected at the Warwick Photoemission Facility, University of Warwick. The samples investigated in this study were attached to electrically-conductive carbon tape, mounted on to a sample bar and loaded in to a Kratos Axis Ultra DLD spectrometer which possesses a base pressure of $\sim 2 \times 10^{-10}$ mbar.

XPS measurements were performed in the main analysis chamber, with the sample being illuminated using a monochromated $\mathrm{Al} \mathrm{K} \alpha$ X-ray source. The measurements were conducted at room temperature and at a take-off angle of $90^{\circ}$ with respect to the surface parallel. The core level spectra were recorded using a pass energy of $20 \mathrm{eV}$ (resolution approx. $0.4 \mathrm{eV}$ ), from an analysis area of 300 microns $\times 700$ microns.
The spectrometer work function and binding energy scale were calibrated using the Fermi edge and $3 \mathrm{~d}_{5 / 2}$ peak recorded from a polycrystalline $\mathrm{Ag}$ sample prior to the commencement of the experiments. The data was analyzed in the CasaXPS package.

Hydrophobic properties were measured using a sessile drop technique, with a video-based optical contact angle measuring device (Attension Theta Optical Tensiometer). Droplet volumes of $6 \mu \mathrm{l}$ of Milli-Q water were dispensed under atmospheric conditions for static contact angle measurements. Angles were calculated by analyzing droplet images recorded on the surfaces after stabilization. Posterior to the measurement, samples were cleaned by absorbing the droplet with a cloth. The as-received, non-laser processed Al2024 surface showed a SCA of $94.63^{\circ} \pm 3^{\circ}$.

\section{Results and discussion}

\section{Microstructure analysis}

Cell like structures were patterned on Al2024 samples using the parameters reported in Table 1. Two different scanning speeds were used. It was important to comprehend that for lower scanning speed, the number of pulses per spot was higher, therefore there was a higher amount of accumulated energy on the surface. The repetition rate was fixed at $100 \mathrm{kHz}$ and a laser spot diameter of $35 \mu \mathrm{m}$ was measured for the given frequency. In order to calculate the accumulated energy on the surface, the number of pulses and the overlapping factor were estimated and presented on Table 2 .

$$
\mathrm{OF}=\frac{2}{\pi D}\left\{D \arccos \left(\frac{d}{D}\right)-d \sqrt{1-\left(\frac{d}{D}\right)^{2}}\right\}
$$

The overlapping factor (OF), illustrated in Fig. 1, can be calculated using eqn (1), where $d=V_{\mathrm{c}} / f_{\mathrm{p}}$ with $V_{\mathrm{c}}$ as the scan speed, and $f$ as the frequency. For a fixed repetition rate of $100 \mathrm{kHz}$, using $100 \mathrm{~mm} \mathrm{~s}^{-1}$ scan speed, the number of pulses per spot was 35 , with a calculated pulse overlap of $96.4 \%$. When scan speed was modified to $40 \mathrm{~mm} \mathrm{~s}^{-1}$, the number of pulses per spot increased to 87 , with $98.5 \%$ of calculated pulse overlap.

Through SEM analysis, as depicted in Fig. 2 one can confirm that micro cell topography was achieved. As the center of the pulsed area was vaporized and melted, it created a channel, and 
Table 2 Structural dimensions of samples patterned with the selected process parameters

Scan speed, $V\left(\mathrm{~mm} \mathrm{~s}^{-1}\right)$

No. of pulses (units)/OF (\%)

Laser fluence, $F\left(\mathrm{~J} \mathrm{~cm}^{-2}\right)$

Laser accumulated fluence $\left(\mathrm{J} \mathrm{cm}^{-2}\right)$

Range of channel depth $(\mu \mathrm{m})$

Range of pillar height $(\mu \mathrm{m})$

Range of pillar width $(\mu \mathrm{m})$

$\begin{array}{ll}100 & \\ 35 / 96.4 \% & \\ 1.56 & 2.08 \\ 42.83 & 57.11 \\ 2.5-4.5 & 9.4-9.8 \\ 3.3-3.9 & 6.5-7.9 \\ 6.7-7.2 & 8.4-9.2\end{array}$

40

$87 / 98.5 \%$

1.56

106.79

Not measured

$6.2-8.3$

$8.5-9.7$
2.08

142.38

2.8-3.4

$7.8-8.0$

$5.6-10.5$ on its edges pillars were formed due to the accumulation of ejected material. Through CLSM analysis, the dimensions of the patterned micro cells were measured and reported on Table 2. However, measurements of depth profile were affected by an observed phenomena, as inside the channels another formation of holes can be detected, increasing deviation on the depth values.

During the laser process, when the laser fluence was changed from 1.56 to $2.08 \mathrm{~J} \mathrm{~cm}^{-2}$, one can observe an increment on the amount of melted and ejected material, correlated by higher structural dimensions.

Also, additional splashed material was detected, with a greater presence of spherical shaped particles, resulting from the resolidification of metal vapor present during the ablation.

Fig. 3 shows SEM images of patterns made with $40 \mathrm{~mm} \mathrm{~s}^{-1}$ scan speed, laser fluence of 1.56 and $2.08 \mathrm{~J} \mathrm{~cm}^{-2}$ and hatch distances of 50 and $100 \mu \mathrm{m}$. Once more, micro cell structures were formed. Though, one can perceive that reducing scan speed enlarged the amount of blasted material.
Patterning with a laser fluence of $1.56 \mathrm{~J} \mathrm{~cm}^{-2}$, the presence of recast near the channels was too high, therefore it was impossible to measure the depth of the channels, as there was a large presence of material on top. Using $2.08 \mathrm{~J} \mathrm{~cm}^{-2}$ of fluence, channels were partially filled with molten material after ablation, thus measured depth values were much lower than those observed for the same energy but with $100 \mathrm{~mm} \mathrm{~s}^{-1}$ of scan speed. It was observed that for a $50 \mu \mathrm{m}$ hatch distance the ejected material started to redeposit in adjacent walls.

For a fixed repetition rate of $100 \mathrm{kHz}$ and a laser spot diameter of $35 \mu \mathrm{m}$, it was important to understand the significance of varying scan speed. As depicted in Fig. 2 and 3, the change of scan speed significantly affected structural sizes and in order to study this effect it was necessary to analyse the accumulated laser fluence ${ }^{33}$ that was being irradiated on the surface.

$$
F_{0}^{\mathrm{tot}}=F_{0} \times \frac{\mathrm{OS}}{1-\mathrm{OF}} \text { so } F_{0}^{\mathrm{tot}} \propto \frac{F_{0} \times f}{V_{\mathrm{s}}}
$$
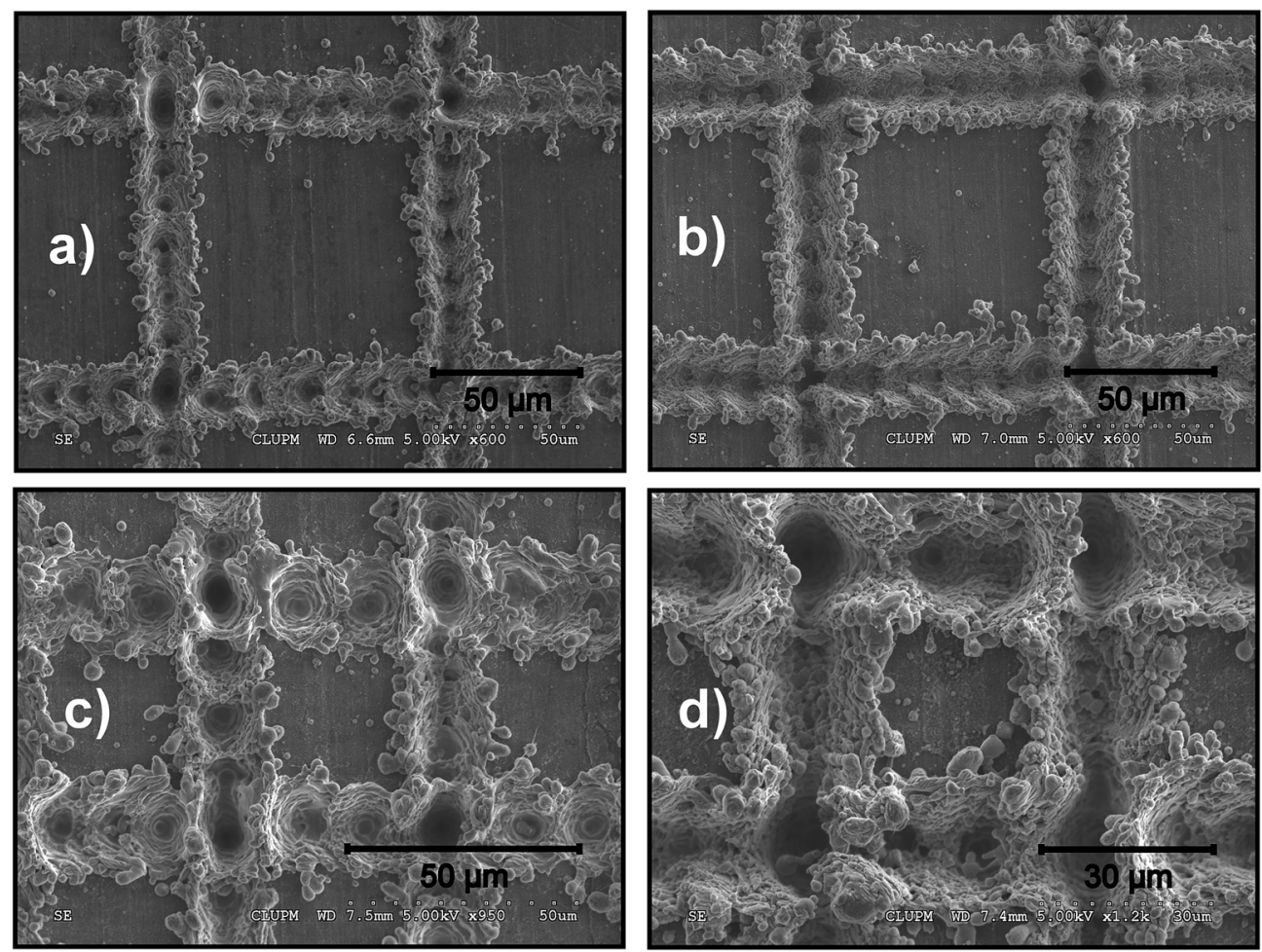

Fig. 2 SEM images of micro cells patterned with $100 \mathrm{~mm} \mathrm{~s}^{-1}$ scan speed, hatch distance of $100 \mu \mathrm{m}$ (top row): laser fluence $1.56 \mathrm{~J} \mathrm{~cm}^{-2}$ (a) and $2.08 \mathrm{~J} \mathrm{~cm}^{-2}$ (b); and with a hatch of $50 \mu \mathrm{m}$ (bottom row): laser fluence $1.56 \mathrm{~J} \mathrm{~cm}^{-2}$ (c) and $2.08 \mathrm{~J} \mathrm{~cm}^{-2}$ (d). 

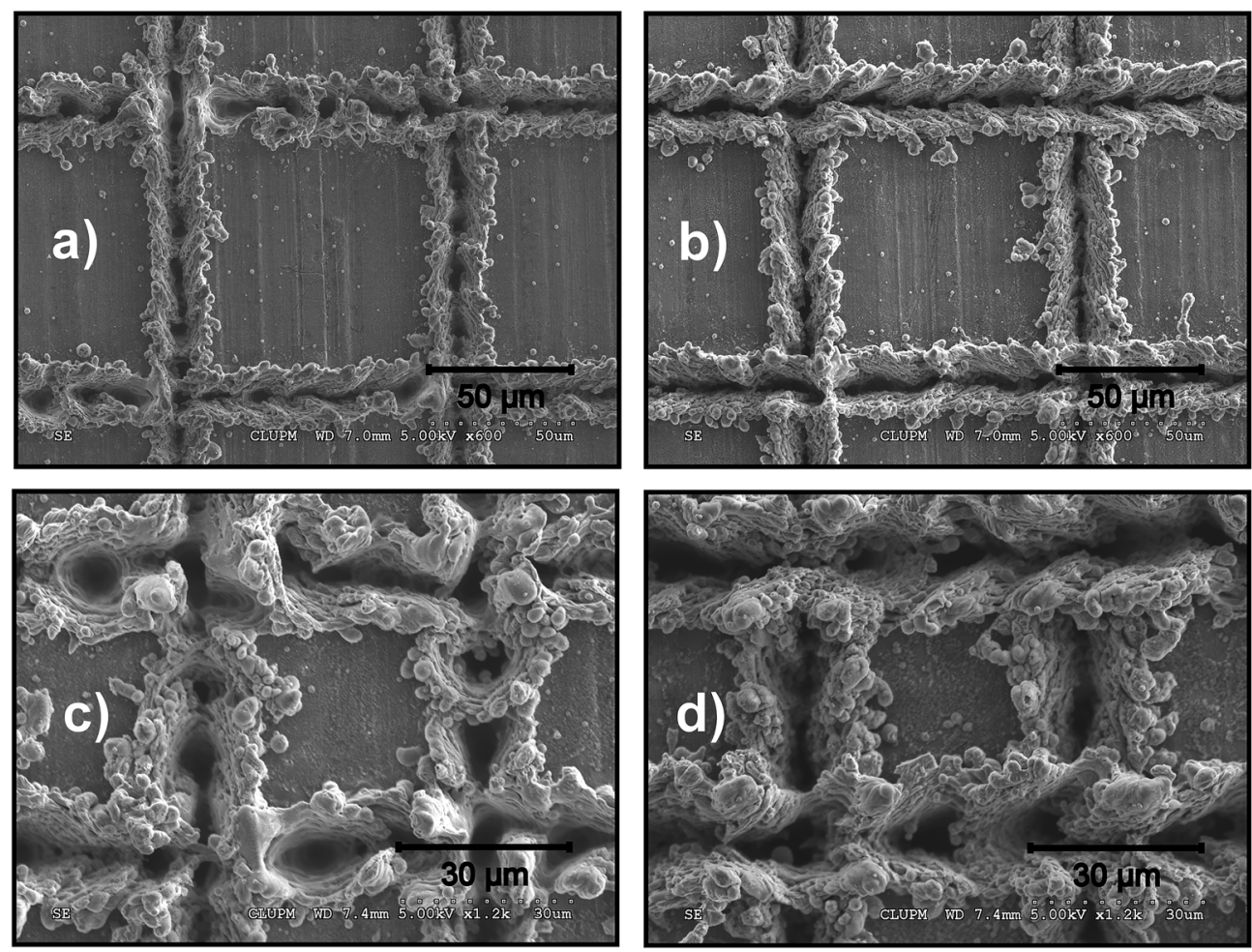

Fig. 3 SEM images of micro cells patterned with, $100 \mathrm{kHz}$ of repetition rate, $40 \mathrm{~mm} \mathrm{~s}^{-1}$ scan speed, hatch distance of $100 \mu \mathrm{m}$ (top row): laser fluence $1.56 \mathrm{~J} \mathrm{~cm}^{-2}$ (a) and $2.08 \mathrm{~J} \mathrm{~cm}^{-2}$ (b); and with a hatch of $50 \mu \mathrm{m}$ (bottom row): laser fluence $1.56 \mathrm{~J} \mathrm{~cm}^{-2}$ (c) and $2.08 \mathrm{~J} \mathrm{~cm}{ }^{-2}$ (d).

$F_{0}^{\text {tot }}$ is the total optical accumulated laser fluence, $F_{0}$ the pulse fluence, OS the number of overscans (for a single scan, $\mathrm{OS}=1$ ) and $\mathrm{OF}$ the overlapping factor. As displayed in eqn (2) the total accumulated fluence is then directly proportional to the pulse fluence $F_{0}$ and repetition rate $f_{\mathrm{p}}$ and has an inverse proportionality with the scan speed $V_{\mathrm{s}}$.

Fig. 4 shows a topography profile obtained using CLSM illustrating the measures of pillar height $(H)$, width $(W)$ and channel depth $(D)$. In order to quantify the amount of recast

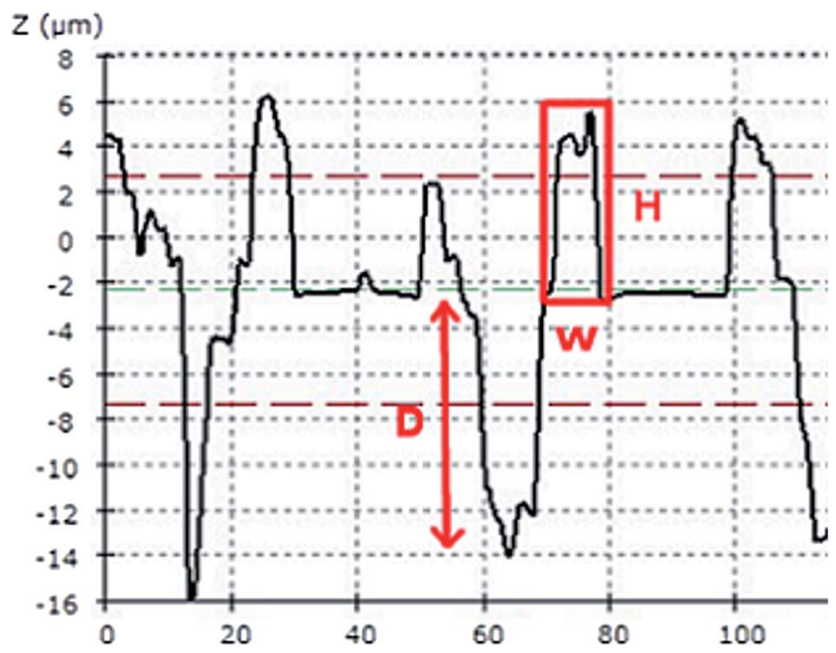

Fig. 4 Topography cross section profile illustrating measurements of height and width of pillars utilized for calculating recast area. material obtained, the cross-section of pillar area was approximated to the area of a rectangle.

Measured values for pillar area are shown in Table 3. Fig. 5 displays a relation between pillar area and accumulated laser fluence.

For the lowest calculated fluence, samples were less ablated, thus less material was removed from the surface and there was few recast formation. For 107 and $142 \mathrm{~J} \mathrm{~cm}^{-2}$ of accumulated laser fluence, the amount of ablated material was higher but there was less control on the shape of the structures, due to an excess of energy. The amount of molten material that was pushed to the channel's sides was so high that the pillars were widened and part of the material slided back inside the channels. Nevertheless, the formed micro cells on the samples' surface were able to trap small volumes of air when in contact with a water droplet, thus increasing hydrophobic potential.

Table 3 Recast area $\left(\mu \mathrm{m}^{2}\right)$ for different laser accumulated fluence and hatch distance values

\begin{tabular}{lllrr}
\hline & \multicolumn{4}{c}{ Laser accumulated fluence $\left(\mathrm{J} \mathrm{cm}^{-2}\right)$} \\
\hline Hatch distance $(\mu \mathrm{m})$ & 42.83 & 57.11 & 106.79 & 142.38 \\
40 & 44.93 & 84.74 & 92.81 & 144.27 \\
50 & 26.92 & 67.67 & 70.54 & 82.46 \\
75 & 26.35 & 58.48 & 76.93 & 83.35 \\
100 & 22.41 & 54.43 & 55.96 & 44.12
\end{tabular}




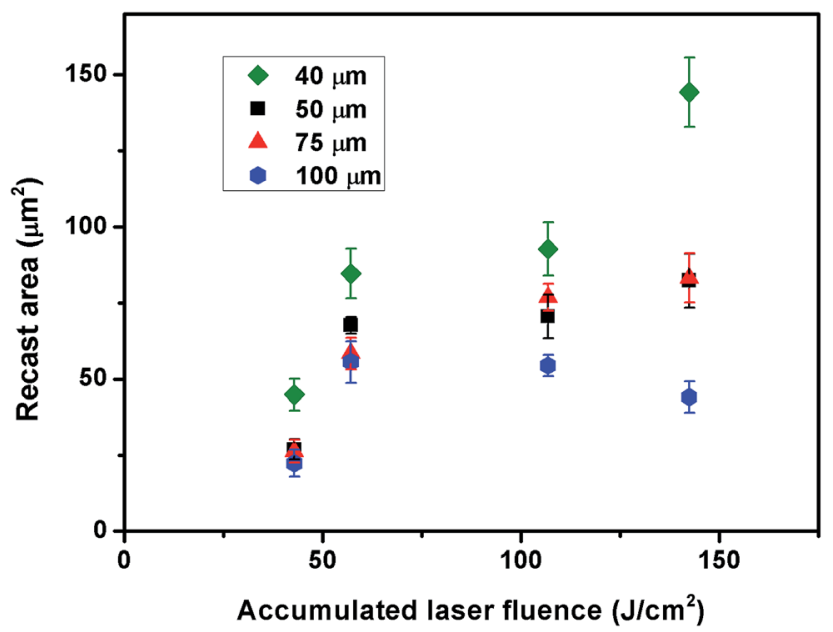

Fig. 5 Comparison of measured recast area for 40, 50, 75 and $100 \mu \mathrm{m}$ hatch distances patterned with different laser accumulated fluence.

\section{Wetting properties}

Initially, once patterned, samples presented a highly hydrophilic behavior, with an immediate spread of the drop once it touched the surface. Bico et al. ${ }^{34}$ reported that after patterning, samples behave like a 3D porous medium as metal oxides are hydrophilic.

The first group of texturized samples was composed of 4 equal Al2024 plates that were each patterned with laser fluence of 1.56 and $2.08 \mathrm{~J} \mathrm{~cm}^{-2}$, scan speed was set to $100 \mathrm{~mm} \mathrm{~s}^{-1}$ and the hatch was varied between 40 and $100 \mu \mathrm{m}$. Posterior to laser patterning, samples were stored inside polyethylene bags $\left(\mathrm{C}_{2} \mathrm{H}_{4}\right)_{n}$ and were analyzed in a regular period for 22 days, where after which the SCA values stabilized. To perform the contact angle measurements, one sample was wet in every measurement and the remaining samples were measured in what was defined as a rotational system, where in each measurement a different sample was used, switching between the three remaining samples, in an order such as 1, 2, 3, 1, 2, 3...

Observing Fig. 6 information related to the aging process was retrieved. In the first days of measurement, highlighted by the blue outline, analysing results for the same fluence, one can observe a difference in behavior on the repeatedly wet samples. The Al2024 patterned areas had an increasing contact angle for both laser powers on the repeatedly wet samples. Which is explained by a combined effect between more frequent exposure to ambient air, hydroxylation of the surface ${ }^{35}$ and chemical adsorption of ions naturally present in pure water. ${ }^{36}$ Also, for the patterns that were measured in a rotational system it was observed that hydrophobic properties appear quicker for surfaces irradiated with less accumulated fluence.

Posteriorly to 15 days, all samples presented a similar superhydrophobic behavior. Since observed results showed that samples which were measured in a rotational system depicted a more expressive difference in contact angle evolution in time, these were further analyzed to define which laser patterning parameters could assure the best SCA values and quicker aging process.

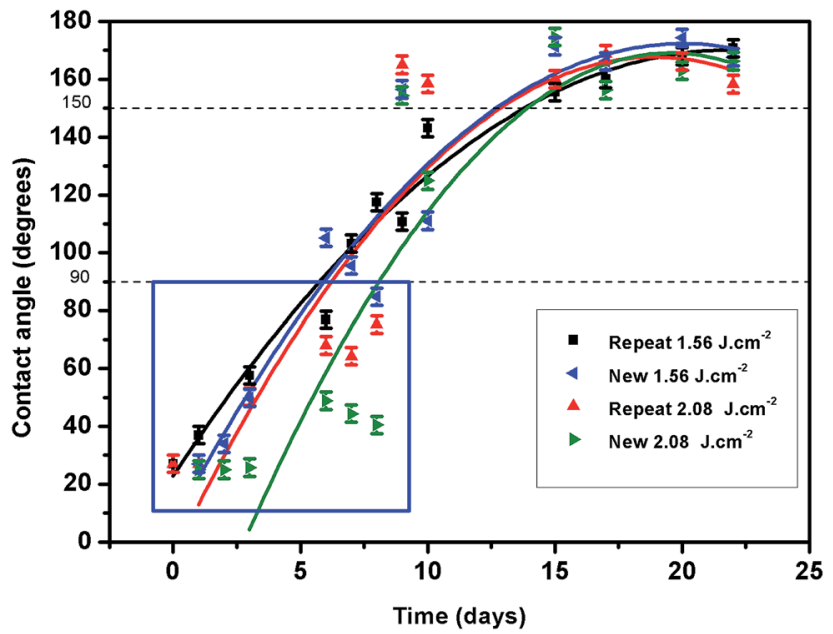

Fig. 6 Comparison of SCA values between samples, patterned with $100 \mathrm{kHz}$ of repetition rate, $100 \mathrm{~mm} \mathrm{~s}^{-1}$ of scan speed, $1.56 \mathrm{~J} \mathrm{~cm}^{-2}$ and $2.08 \mathrm{~J} \mathrm{~cm}^{-2}$ of laser fluence and $40 \mu \mathrm{m}$ of hatch distance, that are consistently tested in every measurement (repeat) and the samples that were measured in a rotational process (new), both stored in polyethylene.

Fig. 7 depicts SCA values for the samples patterned with $1.56 \mathrm{~J} \mathrm{~cm}^{-2}$ and $2.08 \mathrm{~J} \mathrm{~cm}^{-2}$ of laser fluence and 40 and $100 \mu \mathrm{m}$ of hatch distance (i.e. samples with 22.41, 26.92, 82.45 and $67.67 \mu \mathrm{m}^{2}$ pillar area). In this graph, it can be observed that patterns with less pillar area achieved hydrophobic properties quicker. Posterior to 15 days after laser processing, all samples presented superhydrophobicity, however it is important to note that the samples with pillar area of $22.41 \mu \mathrm{m}^{2}$ presented a decay on the final value. Such decay was observed due to this surface $100 \mu \mathrm{m}$ of hatch distance, where large periodicity between structures formed an inefficient air trapping. Also, droplets were exposed to a bigger and hydrophilic non-patterned area, located in the centre of the micro cell, consequently decreasing

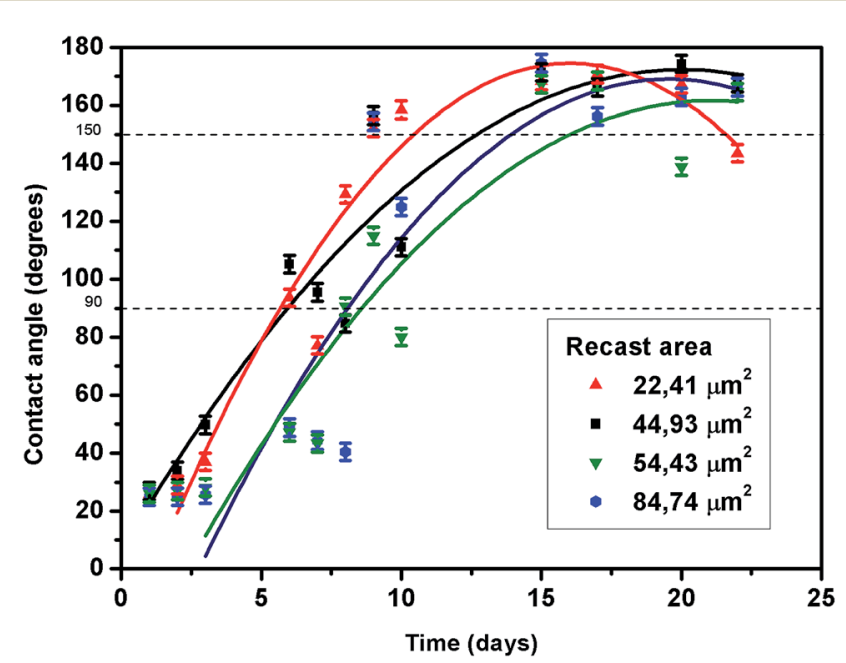

Fig. 7 Time evolution of SCA values of samples with 22.41, 26.92, 82.45 and $67.67 \mu \mathrm{m}^{2}$ pillar area. Patterns were measured in a rotational process and stored in polyethylene. 
the contact angle. Concluding that on a final stage, when SCA values stabilize, the most defining factor is the structure size and gap, confirming results previously reported by Jagdheesh et $a .^{5}$

Posterior to analysing the samples that were kept in plastic polyethylene bags, it was then required to observe the behavior when a different storing condition was used.

Samples were produced with identical sets of patterns in several Al2024 plates that were each kept inside different polystyrene boxes. These were maintained closed in lab environment and were only opened to perform wettability measurements. In every SCA measurement the first two patterns were always repeated and each time two new samples, which were previously never wet, were tested, in order to study the effect of daily wetting the samples on the aging process.

In this study, samples with $100 \mathrm{kHz}$ of repetition rate, $100 \mathrm{~mm} \mathrm{~s}^{-1}$ scan speed, $1.56 \mathrm{~J} \mathrm{~cm}^{-2}$ of laser fluence and 50,75 and $100 \mu \mathrm{m}$ of hatch distance were produced. The latter value was varied to confirm that the behavior was similar for every hatch distance.

Fig. 8 shows the contact angle values for the samples with patterns of $75 \mu \mathrm{m}$ of hatching distance. The results obtained for all hatch distances were similar to the ones depicted and confirm that repeatedly wet samples primarily achieve hydrophobic properties. However, SCA values for non-previously wet samples showed that the aging process was stagnating. Results showed that surfaces presented a random behavior with only one measured hydrophobic sample registered after 37 days of processing. Additionally, it can be observed that hydrophobic properties appear later on these patterns than for the samples stored in polyethylene, which revealed an influence of the sample storage conditions on the aging process.

Observing Fig. 9, it was clear that the amount of recast material influences the appearance of hydrophobic properties.

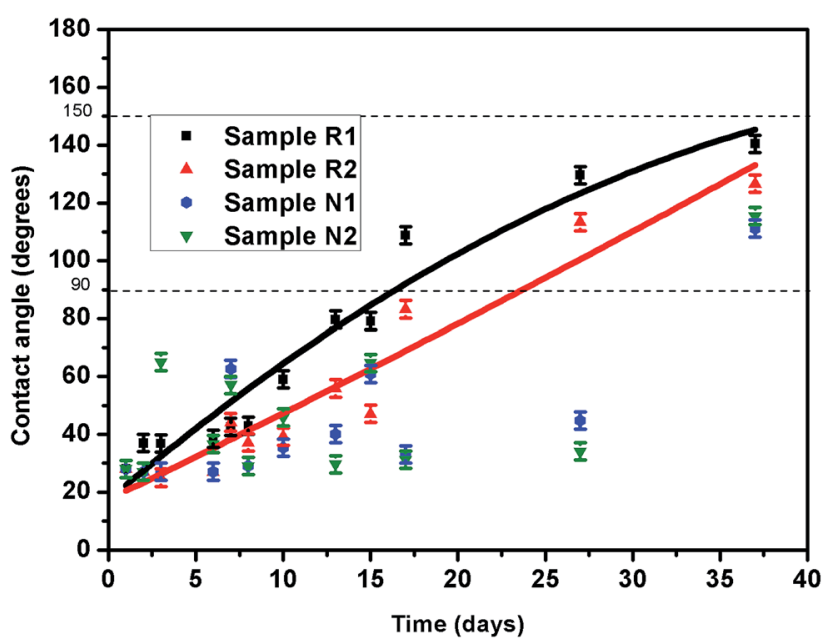

Fig. 8 Time evolution of SCA values of samples patterned with $100 \mathrm{kHz}$ of repetition rate, $100 \mathrm{~mm} \mathrm{~s}^{-1}$ of scan speed, $1.56 \mathrm{~J} \mathrm{~cm}^{-2}$ of laser fluence and $75 \mu \mathrm{m}$ of hatch distance. Displayed in black and red (samples R1 and R2), patterns were repeatedly measured and in blue and green (samples N1 and N2) two new patterns were measured each day, both samples were stored in polystyrene.

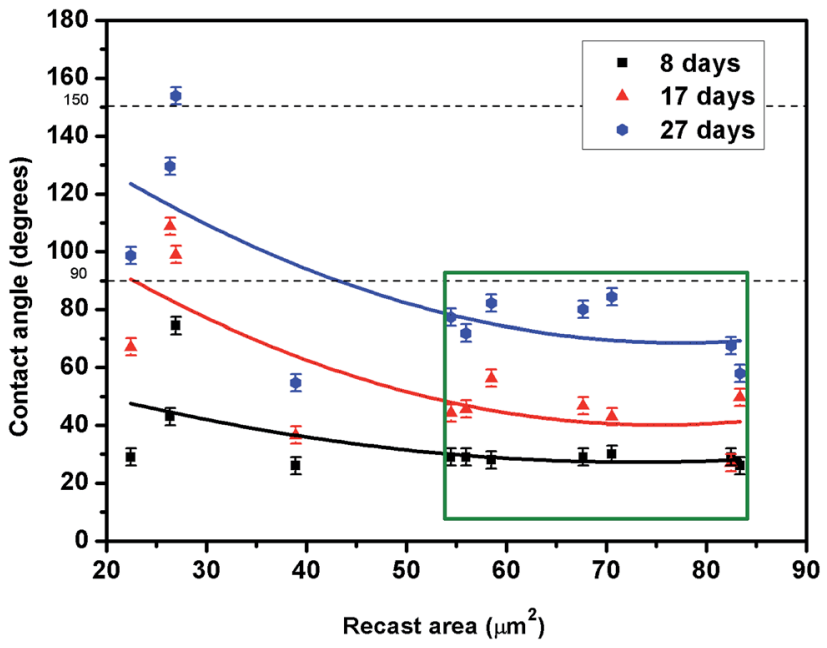

Fig. 9 SCA values measured after 8,17 and 27 days for samples with different recast areas processed with $100 \mathrm{kHz}$ of repetition rate, $100 \mathrm{~mm} \mathrm{~s}^{-1}$ of scan speed, $1.56 \mathrm{~J} \mathrm{~cm}^{-2}$ and $2.08 \mathrm{~J} \mathrm{~cm}^{-2}$ of laser fluence and 50, 75 and $100 \mu \mathrm{m}$ of hatch distance. Patterns were measured every time and samples were stored in polystyrene.

As for the recast area measured above $50 \mu \mathrm{m}^{2}$, even posterior to 27 days samples presented hydrophilic behavior (i.e. highlighted by a green box in Fig. 9), indicating that the use of high fluence combined with small hatch distance was not appropriate to obtain hydrophobicity in $\mathrm{Al} 2024$ samples.

In order to perform an overall comparison on wettability, samples were patterned with laser fluence of 1.56 and $2.08 \mathrm{~J} \mathrm{~cm}^{-2}$, scan speed of 100 and $40 \mathrm{~mm} \mathrm{~s}^{-1}$ and a hatch distance of $50 \mu \mathrm{m}$. After patterning, samples were stored in a laboratory environment exposed to air. Fig. 10 shows the measured contact angle values after 4 and 8 days of processing in relation to recast height. With no special sample storage conditions, superhydrophobic behavior was observed after only 4 days. Confirming previous results, this value was measured for the sample which presented less recast

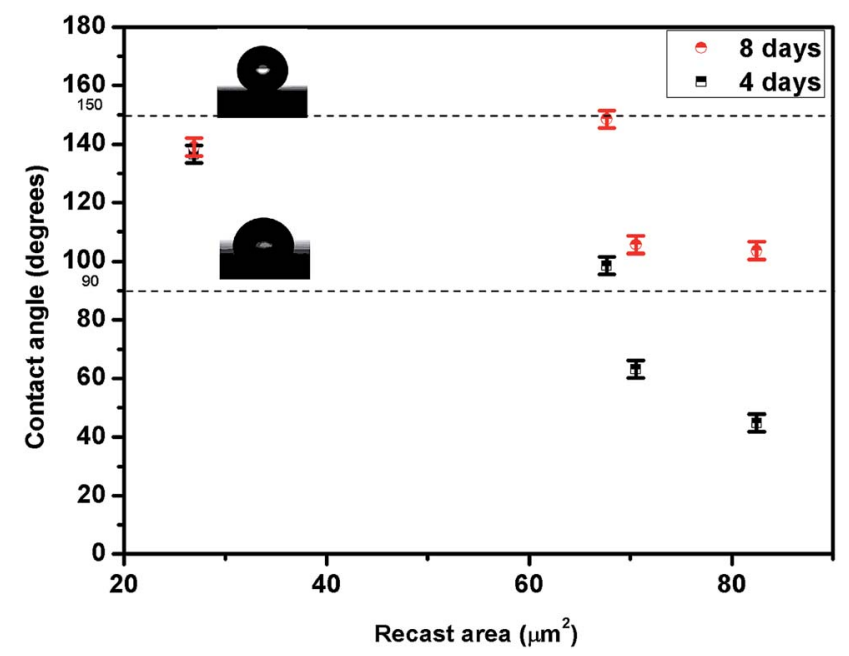

Fig. 10 Comparison of SCA values measured after 4 and 8 days for samples with different recast area that were exposed to ambient air. 
area. This is due to the removal of organic molecules from atmospheric contamination upon laser ablation of the $\mathrm{Al} 2024$ plate. Brand et al. ${ }^{36}$ reported that organic molecules present on aluminium oxide surfaces are not removable with solvents, and thus the non-patterned areas still present hydrocarbons even after cleaning with chemical solvents. When pillars are formed the recast material is composed of new aluminium oxides that are highly hydrophilic. The walls formed by pillars directly interact with the droplets and their high surface energy and polarity attracts water molecules, explaining why samples after patterning presented hydrophilic behavior. On the newly formed aluminium oxide there is a quick adsorption of surface hydroxyls from water molecules present in the air, which constitutes a primary bound to organic functional groups, that are non-polar, have less surface energy and consequently hydrophobic. Therefore when the amount of recast material is higher, or when the pillars are bigger and wider, surface oxide formation is more favourable, exposing a greater area of hydrophilic material that will consequently require a superior amount of time to achieve hydrophobicity. Further analysing Fig. 10, it can be observed that after 8 days, all measured samples were found to be hydrophobic.

Fig. 11 depicts the comparison of results obtained for samples patterned with $100 \mathrm{kHz}$ of repetition rate, $100 \mathrm{~mm} \mathrm{~s}^{-1}$ of scan speed laser fluence of $1.56 \mathrm{~J} \mathrm{~cm}^{-2}$. Analysing the data, one can observe that the sample storage condition was an impact factor in the time evolution of contact angles. Samples stored in a laboratory environment, exposed to air, presented hydrophobic properties prior to the remainder. Though the sample stored in polyethylene was patterned with $40 \mu \mathrm{m}$ and therefore had higher recast area than the remainder, results depicted in Fig. 7 for different recast areas show that the aging process was delayed, when compared to exposure to air, as samples presented hydrophobic behavior after 7 days of

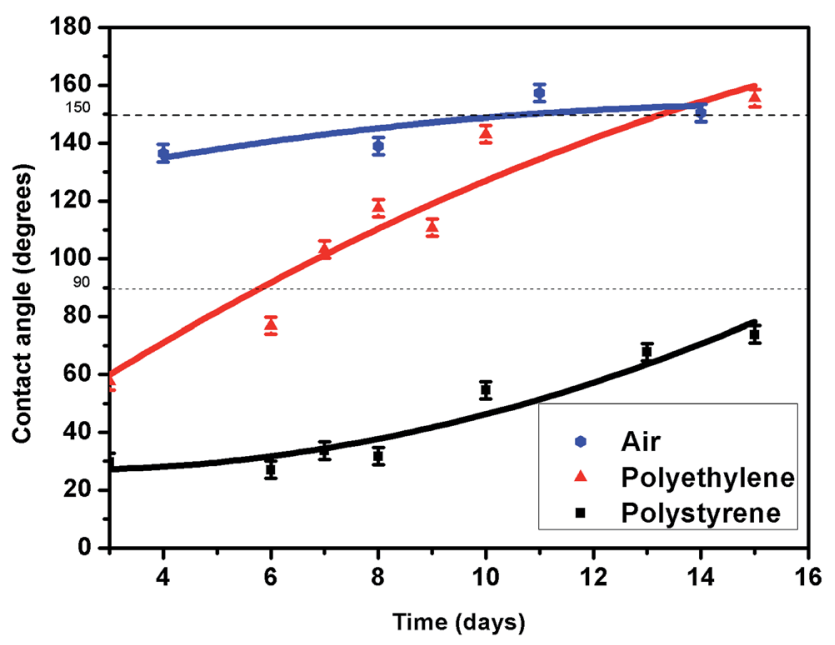

Fig. 11 Comparison of SCA values of samples patterned with $100 \mathrm{kHz}$ of repetition rate, $100 \mathrm{~mm} \mathrm{~s}^{-1}$ of scan speed, laser fluence of $1.56 \mathrm{~J} \mathrm{~cm}^{-2}, 40 \mu \mathrm{m}$ of hatch distance for the sample stored in polyethylene bag and $50 \mu \mathrm{m}$ for the samples kept inside a polystyrene box and air. Samples depicted for all conditionings were wet and measured every day. processing. Subsequently, the sample stored in a polystyrene box did not achieve hydrophobic properties for a 15 day span. These dissimilarities are explained by the surfaces' more frequent contact with air. Samples that were kept in polystyrene were stored with a reduced volume of air inside. Adsorption of water vapour and organic molecules present in the environment was minimized as air flow was reduced but still present due to the non-hermetic packaging. Samples inside polyethylene bags where sealed with a ziplocker, thus also not guaranteeing an airtight packaging, allowing for airflow. Additionally, final SCA values for the polystyrene box samples did not reach values as high as the ones presented for the samples stored in bags and air. Even after 37 days of processing the values were below superhydrophobic, with measured SCA values of $140.52^{\circ}$, much lower than the $170.73^{\circ}$ obtained in 22 days for samples stored in polyethylene. Hence, storage conditions were proved to be a defining factor in the aging process, mainly because of the presence of airflow, as the samples exposed to the ambient air were the fastest to achieve hydrophobic properties. The different behaviour between the polyethylene and polystyrene conditioning is also mainly related to a more efficient air seal by the polystyrene box and a more constant exposure of the polyethylene bag samples to ambient air and potential environmental contamination while performing SCA measurements. Additionally, since polystyrene has a longer organic chain $\left(\mathrm{C}_{8} \mathrm{H}_{8}\right)_{n}$ than the low-density polyethylene $\left(\mathrm{C}_{2} \mathrm{H}_{4}\right)_{n}$ present in the bags, due to structural defects on the polymer, large pores are present in a much higher concentration, so polystyrene is more prone to absorb and retain organic molecules from the atmosphere. ${ }^{37,38}$ As both polymers are non polar, they absorb water in minimal quantities and constitute a good barrier to it, ${ }^{39}$ considering that the polystyrene boxes were much thicker than the polyethylene bags, the combination of the previously described factors with these conditions, led to a stagnated aging process for the samples that were stored in polystyrene.

\section{Surface chemistry}

The second reason for superhydrophobicity transition was reported by Long et al. ${ }^{23}$ to be surface chemistry changes on metal oxides. To understand the behavior of $\mathrm{Al} 2024$ posterior to the laser processing, XPS measurements were performed on three samples: a reference non patterned sample, a sample which was processed in the day before and stored in a polystyrene box, therefore hydrophilic, and a superhydrophobic sample. Patterns on the surfaces were made with $100 \mathrm{kHz}$ repetition rate, $1.56 \mathrm{~J} \mathrm{~cm}^{-2}$ fluence, $100 \mathrm{~mm} \mathrm{~s}^{-1}$ of scan speed and $40 \mu \mathrm{m}$ of hatch. Fig. 12 depicts the spectra for the measured samples. By observing the displayed results one can detect the presence of three major contributions, O 1s, C 1s and Al 2p. Minimal atomic percentages were measured for the other elements that compose the alloy, therefore those were not considered to play an important role in the surface chemistry. Analysis of the binding energies of these peaks revealed that aluminium was mainly present in $\mathrm{Al}_{2} \mathrm{O}_{3}$ form, commonly known as alumina, additionally carbon was detected through the presence of hydrocarbon organic molecules on the surface. 


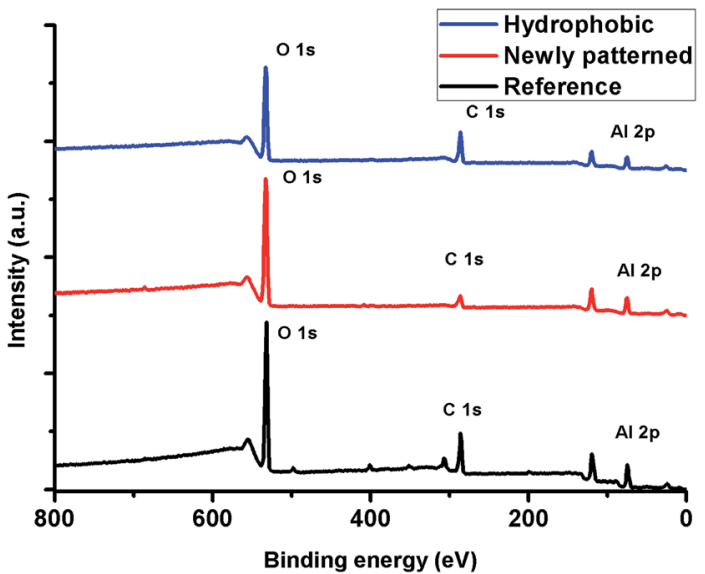

Fig. 12 XPS spectra for a reference non patterned sample (black), a sample patterned in the day before measure (red) and a sample after one month of patterning which presented superhydrophobic behavior (blue).

Table 4 shows composition analysis with atomic percentage quantities of the main peaks measured in the three surfaces.

For the reference sample composed of $\mathrm{Al} 2024$ non patterned surface, there was an obvious presence of aluminium oxide which exhibited an $\mathrm{Al} 2 \mathrm{p}_{3 / 2}$ peak at $75.5 \mathrm{eV}$, an $\mathrm{O} 1 \mathrm{~s}$ peak at $532.3 \mathrm{eV}$, and the correct $\mathrm{O} / \mathrm{Al}$ ratio for the $\mathrm{Al}_{2} \mathrm{O}_{3}$ stoichiometry. Additionally, carbon content was relatively high with a 1.12 ratio over aluminium, this is related with the presence of adsorbed hydrocarbons from the prolonged exposure to ambient air, and that XPS is naturally more sensitive to the outer surface of the material compared to the bulk.

For the hydrophilic sample that was patterned in the day before XPS measurement, it can be observed a noticeable decrease on carbon content. During the laser processing, organic molecules that were present in the original sample were destroyed by the beam incidence. Once pillars were formed by the recast accumulation, there was a rapid passivation on the surface, creating an outer layer composed of $\mathrm{Al}_{2} \mathrm{O}_{3}$. Through the formation of the oxide layer, atoms of $\mathrm{Al}$ and $\mathrm{O}$ organized on a lattice to form alumina. ${ }^{40}$ However, a considerable amount of atoms were not immediately paired, these unsaturated elements, in the form of $\mathrm{Al}^{3+}$ and $\mathrm{O}^{-}$, then constituted Lewis acid and base pairs on the surface of the oxide layer. ${ }^{41}$ Due to the combination of these polar unsaturated atoms, posterior to patterning, the surface free energy was high, causing a hydrophilic behavior.

When the surface was exposed to ambient air, the Lewis acid-base pairs were subjected to hydroxylation from airborne water molecules. ${ }^{5}$ During this reaction adsorbed water vapour

Table 4 Chemical composition of the measured samples

\begin{tabular}{llllll}
\hline & $\mathrm{Al}(\mathrm{at} \%)$ & $\mathrm{O}(\mathrm{at} \%)$ & $\mathrm{C}(\mathrm{at} \%)$ & $\mathrm{C} / \mathrm{Al}$ & $\mathrm{O} / \mathrm{Al}$ \\
\hline Reference & 25.80 & 40.99 & 28.85 & 1.12 & 1.59 \\
New & 28.49 & 51.44 & 16.41 & 0.57 & 1.81 \\
Hydrophobic & 21.30 & 42.50 & 33.85 & 1.59 & 2.00
\end{tabular}

molecules dissociated into $\mathrm{OH}^{-}$and $\mathrm{H}^{+}$then paired with $\mathrm{Al}^{3+}$ and $\mathrm{O}^{-}$, forming $\mathrm{Al}^{3+} \mathrm{OH}^{-}$. This effectively passivated the surface and reduced the strength of the Lewis acid base pairs, further decreasing the total surface energy and consequently lowering the wettability of the samples. Due to a fast hydroxylation, the newly patterned sample, already presented a mixed layer of hydroxides and oxides on the surface, confirmed by the higher O/Al ratio comparing to the reference sample, such results were also reported by Brand et $a .^{36}$ and Jagdheesh et al. ${ }^{5}$

Despite the destruction of the organic molecules and the formation of new oxides, carbon is still detected in the hydrophilic sample, due to the presence of non processed areas, along with a quick attachment from few hydrocarbons on the surface.

Since the hydroxylation process is very fast and previous static contact angle measurements also confirmed that hydrophobic properties were progressively obtained when samples were exposed to ambient air, and considering that hydroxides present high affinity to water molecules, it can be derived that hydrophobicity was not directly caused by the hydroxylation.

$$
\mathrm{RCOOH}_{(\mathrm{g})}+\mathrm{Al}-\mathrm{OH} \rightarrow \mathrm{RCOO}-\mathrm{Al}+\mathrm{H}_{2} \mathrm{O}
$$

Consequently, as reported by Brand et al.,${ }^{36}$ hydrophobic properties are obtained when organic molecules, present in the ambient air moisture, attach to the surface. Through interaction with the hydroxide groups created during the hydroxylation process, carboxylates are chemisorbed. Eqn (3) details the chemical reaction that occurred on the surface when samples were exposed to ambient air. ${ }^{\mathbf{4 2}}$ Chemisorbed carboxylates present in air moisture, such as formic and acetic acid, ${ }^{\mathbf{4 3}}$ represent short chain organic molecules that are non polar and therefore lower the total surface free energy. This depolarization of the surface was responsible for the hydrophilic to hydrophobic transition, and explains the reason why samples that were not exposed to ambient air have a slower transition.

XPS results show the highest carbon to aluminium ratio for the superhydrophobic sample, confirming the attachment of carboxylates on the surface. The ratio of oxygen to aluminium was also the highest detected, as the outer layers of the surface were populated by carboxylates and oxyhydroxides. Due to the XPS short depth analysis, which only detects approximately $7 \mathrm{~nm}$ for $\mathrm{C}$ 1s photoelectrons and $8 \mathrm{~nm}$ for $\mathrm{Al} 2 \mathrm{p}$ photoelectrons, aluminium content was the lowest measured value in this sample.

Long et $a .^{23}$ reported that in order to characterize the surface polarity of the samples, not only the value of $\mathrm{C} / \mathrm{Al}$ ratio should be analyzed but also the concentration of $\mathrm{C}-\mathrm{C}(\mathrm{H})$. The combination of these two values gives a more accurate description of the polarity of the surface, as organic chains tend to be longer when the $\mathrm{C}-\mathrm{C}(\mathrm{H})$ concentration is high. XPS measurements allowed to quantify the percentage of this bond. Obtained results were $68.57 \%, 40.66 \%$ and $31.68 \%$, for the reference, hydrophobic and hydrophilic sample respectively. Percentage values of bond concentrations suggest that over the course of time, samples that were exposed to ambient air tend to increase the chain length of organic species. Although, the hydrophobic sample possesses the 
highest concentration of carbon content the medium value of $\mathrm{C}$ $\mathrm{C}(\mathrm{H})$ bond percentage indicates that primarily there was chemisorption of short chained organic molecules. ${ }^{44}$

It has been reported by several authors that an organic rich environment increases the speed of the aging process. ${ }^{23,36,45}$ So, an elevated atmosphere maximizing the presence of organic molecules would promote a faster aging process. However, posterior to the hydroxylation process, the presence of water vapor molecules in the atmosphere stimulates the adsorption of a second layer of non-dissociated water molecules through the formation of hydrogen bonds with the preadsorbed hydroxyl groups present on the hydroxylated surfaces of oxides, which hinders the further adsorption of hydrocarbons, ${ }^{28,36,46}$ since these reactive sites are passivated. Jagdheesh et al. ${ }^{47}$ reported that when using a vacuum process, lubricating oils present in the pumping mechanisms may release organic molecules into the vacuum chamber and contaminate the sample. Consequently there is a more efficient adsorption of hydrocarbons, due to the very low partial pressure of water vapor that can be reached at high vacuum. Future investigations on the influence of a vacuum assisted system in the aging process of $\mathrm{Al} 2024$ will be performed.

\section{Conclusions}

Combining surface chemistry changes and micro cell patterning, superhydrophobic samples were developed on Al2024 aluminium alloy with a cost efficient nanosecond infrared laser.

Wettability measurements were performed and the results demonstrated that surfaces with superhydrophobic properties were created without any post treatment. Storage conditions were found to have a significant influence on SCA evolution in time due to the reduced airflow. The accumulated laser fluence had a direct impact on the dimensions and shape of micro structures, i.e. micro pillars, formed by the ablation process, and they were found to influence the aging process. The results showed that the volume of ejected material that formed the micro pillars had an inverse relation with the rate of hydrophobic properties appearance.

XPS measurements were carried out and confirmed that surface chemical changes influence the wettability of the samples. Organic molecules responsible for low surface energy and a nonpolar surface were detected through a higher concentration of carbon on the superhydrophobic sample, confirming molecule adsorption from aluminium oxides present on the surface posterior to patterning.

\section{Conflicts of interest}

There are no conflicts to declare.

\section{Acknowledgements}

This work was carried out in the framework of the LASER4FUN project (http://www.laser4fun.eu), which has received funding from the European Union's Horizon 2020 research and innovation programme under the Marie Skłodowska-Curie grant agreement No. 675063.

\section{References}

1 D. V. Ta, A. Dunn, T. J. Wasley, R. W. Kay, J. Stringer, P. J. Smith, C. Connaughton and J. D. Shephard, Appl. Surf. Sci., 2015, 357, 248-254.

2 A. Marmur, Langmuir, 2004, 20, 3517-3519.

3 R. Jagdheesh, B. Pathiraj, E. Karatay, G. R. B. E. Römer and A. J. Huis in't Veld, Langmuir, 2011, 27, 8464-8469.

4 A. A. Chaudhari, J. Coppage-Gross, K. K. Rangan, V. C. Onyilo, T. S. Sudarshan, S. R. Singh and S. R. Pillai, Mater. Manuf. Processes, 2016, 31, 1156-1161.

5 R. Jagdheesh, M. Diaz and J. L. Ocana, $R S C A d v$., 2016, 6, 72933-72941.

6 S. Nishimoto and B. Bhushan, RSC Adv., 2012, 671-690.

7 D. Lv, J. Ou, M. Xue and F. Wang, Appl. Surf. Sci., 2015, 333, 163-169.

8 N. Wang, D. Xiong, M. Li, Y. Deng, Y. Shi and K. Wang, Appl. Surf. Sci., 2015, 355, 226-232.

9 R. Jagdheesh, B. Pathiraj, A. G. Marin, D. A. Cerro, R. G. H. Lammertink, D. Lohse, A. J. Huis and G. R. B. E. Römer, Phys. Fluids, 2010, 1-6.

10 A. M. Kietzig, S. G. Hatzikiriakos and P. Englezos, Langmuir, 2009, 25, 4821-4827.

11 B. Wu, M. Zhou, J. Li, X. Ye, G. Li and L. Cai, Appl. Surf. Sci., 2009, 256, 61-66.

12 K. Wang, Y. Dong, Y. Yan, S. Zhang and J. Li, RSC Adv., 2017, 7, 29149-29158.

13 S. A. Kulinich and M. Farzaneh, Surf. Sci., 2004, 573, 379390.

14 M. Nosonovsky and B. Bhushan, Ultramicroscopy, 2007, 107, 969-979.

15 A. M. Escobar and N. Llorca-Isern, Appl. Surf. Sci., 2014, 305, 774-782.

16 J. Tan, J. Hao, Z. An and C. Liu, $R S C A d v ., 2017$, 7, 2614526152.

17 Y. Zhang, D. Ge and S. Yang, J. Colloid Interface Sci., 2014, 423, 101-107.

18 C. Momma, B. N. Chichkov, S. Nolte, F. vonAlvensleben, A. Tunnermann, H. Welling and B. Wellegehausen, Opt. Commun., 1996, 129, 134-142.

19 R. Jagdheesh, J. J. García-Ballesteros and J. L. Ocaña, Appl. Surf. Sci., 2016, 374, 2-11.

20 A. B. D. Cassie and S. Baxter, Trans. Faraday Soc., 1944, 40, 546-550.

21 A. M. Peters, C. Pirat, M. Sbragaglia, B. M. Borkent, M. Wessling, D. Lohse and R. G. H. Lammertink, Eur. Phys. J. E, 2009, 29, 391-397.

22 D. Kim, J. Gil and C. Nam, Mater. Lett., 2016, 170, 18-20.

23 J. Long, M. Zhong, H. Zhang and P. Fan, J. Colloid Interface Sci., 2015, 441, 1-9.

24 I. Kiper, R. Fulcrand, C. Pirat, G. Simon, B. Stutz and S. M. M. Ramos, Colloids Surf., A, 2015, 482, 617-623.

25 A. Esmaeilirad, M. V Rukosuyev, M. B. G. Jun and F. C. J. M. Van Veggel, Surf. Coat. Technol., 2016, 285, 227234. 
26 Z. Yoshimitsu, A. Nakajima, T. Watanabe and K. Hashimoto, Langmuir, 2002, 18, 5818-5822.

27 H. J. Choi, J. H. Shin, S. Choo, S. W. Ryu, Y. D. Kim and H. Lee, Thin Solid Films, 2015, 585, 76-80.

28 S. Takeda, M. Fukawa, Y. Hayashi and K. Matsumoto, Thin Solid Films, 1999, 339, 220-224.

29 M. Rahimi, P. Fojan, L. Gurevich and A. Afshari, Appl. Surf. Sci., 2014, 296, 124-132.

30 A. Kietzig and M. N. Mirvakili, J. Adhes. Sci. Technol., 2011, 25, 2789-2809.

31 S. Moradi, S. Kamal, P. Englezos and S. G. Hatzikiriakos, Nanotechnology, 2013, 24, 415302.

32 M. C. Sharp, A. P. Rosowski and P. W. French, Nanosecond laser texturing of aluminium for control of wettability, ed. M. Green and C. Rose, 2015, p. 96570J.

33 D. Scorticati, G. Römer, D. F. de Lange and B. Huis in't Veld, J. Nanophotonics, 2012, 6, 63528.

34 J. Bico, U. Thiele and D. Quéré, Colloids Surf., A, 2002, 206, 41-46.

35 M. M. Gentleman and J. A. Ruud, Langmuir, 2010, 26, 14081411.

36 J. Van Den Brand, S. Van Gils, P. C. J. Beentjes, H. Terryn and J. H. W. De Wit, Appl. Surf. Sci., 2004, 235, 465-474.
37 J. P. Larmann, J. J. DeStefano, A. P. Goldberg, R. W. Stout, L. R. Snyder and M. A. Stadalius, J. Chromatogr. A, 1983, 255, 163-189.

38 E. C. Baughan, Trans. Faraday Soc., 1948, 44, 495.

39 N. S. Sangaj and V. C. Malshe, Prog. Org. Coat., 2004, 50, 2839.

40 T. Bauer, T. Schmaltz, T. Lenz, M. Halik, B. Meyer and T. Clark, ACS Appl. Mater. Interfaces, 2013, 5, 6073-6080.

41 K. C. Hass, W. F. Schneider, A. Curioni and W. Andreoni, Science, 1998, 282, 265-268.

42 S. R. Tong, L. Y. Wu, M. F. Ge, W. G. Wang and Z. F. Pu, Atmos. Chem. Phys., 2010, 10, 7561-7574.

43 E. Dabek-Zlotorzynska and V. Celo, in Capillary Electrophoresis: Methods and Protocols, ed. P. SchmittKopplin, Humana Press, Totowa, NJ, 2008, pp. 43-64.

44 M. R. Alexander and T. M. Duc, J. Mater. Chem., 1998, 8, 937943.

45 A.-M. Kietziga, M. N. Mirvakilia, S. Kamalb, P. Englezosa and S. G. Hatzikiriakosa, J. Adhes. Sci. Technol., 2011, 25, 12931303.

46 M. Psarski, J. Marczak, J. Grobelny and G. Celichowski, J. Nanomater., 2014, 2014, 1-11.

47 R. Jagdheesh, M. Diaz, S. Marimuthu and J. L. Ocana, J. Mater. Chem. A, 2017, 5, 7125-7136. 